\title{
FUNDAMENTAL PARAMETERS OF CP STARS
}

\author{
P. NORTH \\ Institut d'Astronomie de l'Université de Lausanne \\ CH-1290 Chavannes-des-Bois, Switzerland
}

\section{Introduction}

The quest for accurate fundamental parameters $\left(M, R, T_{\mathrm{eff}}, \log g, v \sin i\right)$ of CP stars has been a lasting problem ever since the nature and origin of these stars has been discussed. The main reason for the difficulty lies in the anomalous colours of these stars, linked with their abundance anomalies which are responsible for complicated blocking and backwarming effects. In the following, we review the $T_{\text {eff }}$ determinations proposed so far, and show how the Hipparcos results improve the radius and $\log g$ determinations of nearby Ap stars.

\section{2. $T_{\text {eff }}$ Determinations}

Because of the UV-flux deficiency due to enhanced line blocking, the $T_{\text {eff }}$ determination is especially delicate for CP2 (magnetic) stars: their visual energy distribution mimicks normal stars with a larger effective temperature, at least when $T_{\mathrm{eff}} \geq 9500 \mathrm{~K}$. The various methods are the following:

\subsection{MORE OR LESS FUNDAMENTAL METHODS}

Code et al. (1977) have provided the most fundamental method, measuring the angular diameter by interferometry and combining it with $m_{b o l}$. The latter requires UV and IR data which are not always available, but must be if a truly fundamental determination is wanted. There is no CP star in their list, except Sirius, a hot Am star. The IR Flux Method (IRFM) first proposed by Blackwell \& Shallis (1977) has been applied to a few tens of CP2 stars (see Mégessier 1988, Glushneva 1987), but its accuracy is often limited by the UV flux uncertainties (old TD1 data have sometimes to be used) and by problems in the absolute flux calibration of the J, H, K, L, M magnitudes. Stepien \& Dominiczak (1989) proposed to define a "model" effective temperature $T_{\mathrm{M}}$ by fitting an energy distribution of a solar-composition model to the observed energy distribution in the visual, and to apply a correction $\Delta_{1}$ to $T_{M}$, which takes the UV flux deficiency into account. Since $\Delta_{1}$ correlates with $T_{\mathrm{M}}$, the knowledge of the visual energy distribution alone is enough, at least for statistical purposes. Detailed modelling of the visual energy distribution observed by spectrophotometry and $\mathrm{H}_{\gamma}$ line profile (Adelman et al. 1995). Using model atmospheres where the real abundance pattern of the star is included, the UV-flux deficiency is taken into account in a natural way, as far as the models are realistic. The $\mathrm{H}_{\gamma}$ profile provides further constraints on $T_{\text {eff }}$ and possibly $\log g$.

\subsection{PHOTOMETRIC METHODS}

They must in any case be calibrated, so they depend necessarily on the preceding, fundamental methods. Let us discuss the Geneva and $u v b y \beta$ photometric systems.

\subsubsection{Geneva System}

Hauck \& North (1993, hereafter HN93) have given the general recipe to obtain $T_{\text {eff }}$ from Geneva colour indices, and the reader should refer to this paper. There are a few modifications, nevertheless: for $\mathrm{CP} 1$ (Am) stars, one can 
use directly the calibration by Künzli et al. (1997), obtaining not only $T_{\text {eff }}$ but also $[M / H]$ if $T_{\text {eff }}<7500 \mathrm{~K}$; the $\log g$ may be biased, however (North et al. 1997). For hot CP2 (Si) stars, the calibration of Künzli et al.

(1997) using the $X$ an $Y$ parancters should be used instead of that by North \& Nicolet (1990); the resulting $T_{\mathrm{M}}(X, Y)$ should then be corrected according to the formula $T_{\mathrm{rff}}=-230+0.941 \times T_{\mathrm{M}}$ (Hauck \& Künzli 1996) which replaces Eqn. 1 of HN93. For cool CP2 (SrCrEu) stars, identified photometrically by $T_{\mathrm{M}}(X, Y) \leq 9700 \mathrm{~K}$, one should use the $B 2-G$ index and Equ. 2 of HN93; in such a case, however, IS reddening must be corrected for, but cannot be obtained from Geneva photometry.

There is a good overall agreement between these photometric $T_{\text {eff }}$ and more fundamental determinations published in the recent literature for CP2 stars: $T_{\text {eff }}($ Geneva $)=1.001 \times T_{\text {eff }}($ litt. $)-87$, with $\sigma($ resid. $)=227 \mathrm{~K}$. The sources of $T_{\text {eff }}$ are Stepien (1994) and references therein, Faraggiana \& Gerbaldi (1993), Hiesberger et al. (1995), Kuschnig et al. (1995), Kupka et al. (1996), Monier (1992), Monier \& Mégessier (1992), Ryabchikova et al. (1995) and Wade (1997).

\subsection{2. uvby $\beta$ System}

For hot CP2 stars, Stepien (1994) proposed to use the reddening-free $[u-b]$ parameter: $\theta_{\mathrm{eff}} \equiv$ $5040 / T_{\text {eff }}=0.200+0.246 \times[u-b]$ for $[u-b] \leq 1.3$ (or $T_{\text {eff }} \geq 9700 \mathrm{~K}$ ). This calibration seems valid, in fact, down to 9000 or even $8500 \mathrm{~K}$. The agreement with $T_{\text {eff }}$ from the literature is fairly good: $T_{\text {eff }}(u v b y \beta)=1.0214 \times T_{\text {eff }}($ litt. $)-223$, with $\sigma$ (resid. $)=303 \mathrm{~K}$. For cool CP2 stars, the calibration of Moon \& Dworetsky (1985) works well, but only below $9000 \mathrm{~K}$.

Interstellar reddening may be determined from the usual calibrations, though less accurately than for normal stars. For hot CP2 stars, Masana et al. (1997) propose a correction to $E(b-y)$ computed from Crawford (1978): $E(b-y)_{C P 2}=E(b-y)_{\text {Craw ford }}-0.001+0.008 \times \Delta p$, where $\Delta p$ is their peculiarity index in the $u v b y \beta$ system.

\section{Radius, mass and $\log g$ determination}

An HR diagram has been established for all CP2, CP3 and CP4 stars with a good Hipparcos parallax $\left(\sigma(\pi) / \pi<0.15\right.$ for $85 \%$ of the sample and $<0.23$ for the whole) and $T_{\text {eff determined with }}$ the IRFM method or spectroscopically. The bolometric correction was taken from Lanz (1984) or Stepien (1994). The visual magnitude has been corrected from three effects: the Lutz-Kelker (1973) bias (modified to take into account the decreasing stellar density away from the galactic plane), duplicity ( $0.75 \mathrm{mag}$ for SB2 and about $\leq 0.2 \mathrm{mag}$ for SB1 binaries) and visual absorption when significant. All stars fall inside the theoretical Main Sequence strip, within the errors. Then, one feels justified in interpolating the mass in the evolutionary tracks of Schaller et al. (1992) for $Z=0.020$ which finally allows to obtain $\log g$ with a typical precision of 0.12 dex. This is estimated from assumed errors $\sigma(\pi) / \pi=0.10, \sigma(B . C)=$.0.1 and $\sigma\left(T_{\text {eff }}\right) / T_{\text {eff }}=0.05$ and is similar to typical errors on photometric estimates for normal stars. Interestingly, the contribution of $\sigma\left(T_{\text {eff }}\right)$ is far from negligible and may widely exceed that of $\sigma(\pi)$.

Another way of obtaining the radius (hence $\log g$ if the mass can be known) is through asteroseismology: knowledge of the frequency spacing $\Delta \nu$ of consecutive overtones for $\mathrm{p}$-modes and of $T_{\text {eff }}$ allows to estimate the absolute magnitude (Martinez 1992). The stars for which $\Delta \nu$ is best known are HD 24712 and HD 60435, whose inferred radii are respectively $1.92 \pm 0.19 R_{0}$ and $2.30 \pm 0.22 R_{\odot}$ (Matthews 1997, private communication). The radii obtained from Hipparcos parallaxes are respectively $1.78 \pm 0.13 \mathrm{R}_{\odot}$ for $T_{\mathrm{eff}}=7330 \pm 140 \mathrm{~K}$ (Wade 1997, HD 24712) and $2.26 \pm 0.60 \mathrm{R}_{\odot}$

for $T_{\text {eff }}=8200 \pm 300 \mathrm{~K}$ (Martinez 1992, HD 60435). The masses interpolated in the evolutionary tracks for the Hipparcos luminosities are $1.600 \pm 0.042 \mathrm{M}_{\odot}$ and $1.97 \pm 0.19 \mathrm{M}_{\odot}$, leading to $\log g=$ $4.08 \pm 0.08$ and $4.01 \pm 0.08$ (asteroseismology), and to $4.14 \pm 0.06$ and $4.02 \pm 0.19$ (Hipparcos) for HD 24712 and 60435 respectively. The agreement is very good.

\subsection{COMPARISON BETWEEN VARIOUS DETERMINATIONS}

A set of hot CP2 (Si type), CP3 and CP4 (He-weak) stars lying closer than 100 po to the sun and having Geneva photometry has been examined. A comparison of the photometric $\log g$ values with those obtained from the Hipparcos parallaxes confirms the results of North \& Kroll (1989): 
for $18 \mathrm{Si}$ stars, the scatter around the regression line is very large $\left(\sigma_{\text {res }}=0.273 \mathrm{dex}\right)$, confirming that the photometric $\log g$ are not reliable. On the other hand, the slope and zero point of the regression line (computed assuming similar errors on both axes) are 1.024 and -0.001 , indicating an overestimate of $\log g$ by photometry of no more than 0.1 dex. For 12 CP 3 stars, on the contrary, the scatter is very small if HD 129174 (a close visual double with composite colours) is excluded, with $\sigma_{\text {res }}=0.080 \mathrm{dex}$. The slope and zero points are 0.654 and 1.398 , so that photometry seems to underestimate high $\log g$ values and overestimate lower ones; but this result should be considered with caution since the dynamics in $\log g$ is no more than 0.5 dex. It is more difficult to conclude regarding the only three $\mathrm{CP} 4$ stars of the sample, whose $\log g$ seems slightly overestimated by the calibration of Geneva photometry.

A comparison has also been attempted between Hipparcos $\log g$ and those obtained from the $\mathrm{H}_{\beta}$ profile of Si stars by North \& Kroll (1989). There are only 6 stars with such a determination and a precise parallax $(\sigma(\pi) / \pi<0.14) .4$ of them are close to the one-to-one relation, but 2 are below it $\left(\log g\left(\mathrm{H}_{\beta}\right)<\log g(\right.$ Hipp. $\left.)\right)$ : HD 147010 and HD 199728. HD 147010 is known as a strongly magnetic star (c.g. Mathys 1995), and its effective temperature - $13080 \mathrm{~K}$, obtained from photometry - might be overestimated, which would imply an overestimate of both the Hipparcos $\log g$ and of that obtained through the $\mathrm{H}_{\beta}$ profile. The magnetic field of HD 199728 is unknown and the reason for the discrepancy remains unclear in both cases. Such a comparison is most interesting in the light of the work by Leblanc et al. (1994) showing that ambipolar diffusion of hydrogen in the presence of a horizontal magnetic field leads to a compression of the stellar atmosphere, so that the effective gravity $g_{\mathrm{eff}}-$ to which the $\mathrm{H}_{\beta}$ profile is sensitive - becomes significantly larger than the purcly gravitational acceleration. However, the reverse behaviour is found here and it remains doubtful that such an effect (which has the advantage of being computed in a self-consistent way) can be detected in this way, unless dramatic improvements in $T_{\text {eff }}$ determinations are achieved.

\section{Conclusion}

Good fundamental parameters are crucial for questions such as the evolution of the magnetic geometry with time (Wade 1997), the difference between roAp and non-oscillating Ap stars (North et al. 1997) and the effect of a strong magnetic field on atmospheric pressure. A very good knowledge of $T_{\text {eff }}$ is extremely important in this context, especially as good $\log g$ values will generally not be obtained quite independently from $T_{\mathrm{eff}}$, wether from Hipparcos or spectroscopic data.

\section{References}

Adelman S.J., Pyper D.M., Lopez-Garcia Z., Caliskan H., 1995, A\&A 296, 467

Blackwell D.E., Shallis M.J., 1977, MNRAS 180, 177

Code A.D., Davis J., Bless R.E., Hanbury-Brown R., 1976, ApJ 203, 417

Crawford D.L., 1978, AJ 83, 48

Faraggiana, R., Gerbaldi M., 1993, in: Peculiar versus normal phenomena in A-type and related stars, eds. M.M. Dworetsky, F. Castelli and R. Faraggiana, ASP Conf. Series 44, 169

Glushneva I.N., 1987, Soviet Astron. 31, 315

Hauck B., Künzli M., 1996, Baltic Astronomy 5, 303

Hauck B., North P., 1993, A\&A 269, 403

Hiesberger F., Piskunov N., Bonsack W.K., Weiss W.W., Ryabchikova. T.A., Kuschnig R., 1995, A\&A 296, 473

Künzli M., North P., Kurucz R.L., Nicolet B., 1997, A\&AS 122, 51

Kupka F., Ryabchikova. T., Weiss W.W., Kuschnig R., Rogl J., Mathys G., 1996, A\&A 308, 886

Kuschnig R., Ryabchikova T., Piskunov N., Weiss W.W., LeContel J.M., 1995, A\&A 294, 757

Lanz. T., 1984, A\&A 139,161

Leblanic F., Michaud G., Babel J., 1994, ApJ 431, 388

Lut $z$ T.E., Kelker D.H., 1973, PASP 85, 573

Martinez P., 1992, in: Variable stars and galaxies, ed. B. Warner, ASP Conf. Series Vol. 30, p. 371

Masana E., Jordi C., Maitzen H.M., Torra. J., 1997, A\&AS, in press

Mathys G., 1995, A\&A 293, 746

Mégessier C., 1988, A\&AS 72, 551

Monier R, 1992, A\&A 263, 175

Monier R., Mégessier C., 1992, A\&A 256, 205

Moon T.T., Dworctsky M.M., 1985, MNRAS 217, 305

North P., Jaschek C., Hauck B., Figueras F., Torra J., Künzli M., 1997, in: Proceedings of the ESA Symposium 'Hipparcos Venice'97', 13-16 May, Venice, Italy, ESA SP-402, p. 239

North P., Kroll R, 1989, A\&AS 78, 325

North P., Nicolet B., 1990, A\&A 228, 78 
Ryabchikova T., Kuschnig R., Piskunov N.E., Pavlova V., 1995, in: Stellar surface structure, IAU Symp. 176, Poster Proceedings, ed. K.G. Strassmeier, p. 132

Schaller G., Schaerer D., Meynet G., Maeder A., 1992, A\&AS 96, 269

Stepien K., 1994, in: Chemically peculiar \& magnetic stars, eds. J. Zverko and J. Ziznovsky, Tatranska Lomnica, Slovak Republic, p. 8

Stepien K., Dominiczak R., 1989, A\&A 219, 197

Wade G.A., 1997, A\&A 325, 1063 